\title{
Los conceptos de patriarcado y androcentrismo en el estudio sociológico y antropológico de las sociedades de mayoría musulmana*
}

\author{
Araceli González Vázquez \\ Collège de France/EHESS. Laboratoire d'Anthropologie Sociale \\ lacuerre@gmail.com
}

Recibido: 10-03-2011

Aceptado: 17-10-2012

\section{Resumen}

En este artículo, realizamos un examen de las conceptualizaciones del patriarcado que mayor influencia ejercen actualmente sobre la forma en que los sociólogos y los antropólogos abordan el estudio de las desigualdades sociales y de género en las sociedades de mayoría musulmana. Igualmente, explicamos el concepto de androcentrismo y evaluamos su fuerza actual como alternativa conceptual y epistemológica frente al concepto de patriarcado.

Palabras clave: antropología; desigualdad social; dominación masculina; países islámicos.

Abstract. The concepts of patriarchy and androcentrism in the sociological and anthropological study of Muslim majority societies

In this article, we examine the theoretical contributions and conceptualizations of patriarchy that have exerted the largest influence on the way sociologists and anthropologists address the study of social and gender inequalities in Muslim majority societies. We also explain the concept of androcentrism and examine its potential as a conceptual and epistemological alternative to the concept of patriarchy.

Keywords: anthropology; social inequality; masculine domination; Islamic countries.

\section{Sumario}

\section{Introducción}

2. La extensión del concepto de patriarcado y la noción de kiriarquía

3. La conceptualización del androcentrismo

4. La influencia del concepto de patriarcado en el estudio de las sociedades de mayoría árabe y musulmana
5. Androcentrismo, ¿alternativa conceptual?, ¿herramienta de visibilización femenina?

6. Referencias bibliográficas

* Este trabajo ha sido realizado gracias a un contrato postdoctoral del programa de formación de investigadores del Gobierno Vasco. 


\section{Introducción}

En su excelente monografía sobre la estructura social, el parentesco y las mujeres en Marruecos, la antropóloga Yolanda Aixelá incluye un epígrafe significativamente titulado: “iPatriarcalidad o androcentrismo?» (2000: 129). En él, además de señalar el modo en que el concepto de patriarcado dificulta la visibilidad de las aportaciones de las mujeres al parentesco y la visibilidad del reconocimiento social que reciben por su participación en la estructura familiar, Aixelá propone hablar de androcentrismo como alternativa conceptual y epistemológica al concepto de patriarcado. La propuesta de Aixelá es relevante, ya que una parte nada desdeñable de la bibliografía sobre las mujeres de las sociedades de mayoría musulmana - particularmente sobre las mujeres marroquíes, objeto de estudio de su tesis doctoral y de la monografía que estamos citando - tiende a reducir el análisis científico a la identificación de las características principales de estas sociedades que nos permiten hablar de «sistema patriarcal», tal y como definen este "sistema» las ciencias sociales. En el caso puntual de Marruecos, la realidad actual —compleja y plural como la de cualquier otro lugar-sugiere que es necesario un mayor énfasis en la revisión de los conceptos, sobre todo porque las investigaciones sociológicas y antropológicas que continúan hablando de una sociedad marroquí contemporánea patriarcal, y también tribal, patrilineal, patrilocal, endogámica, poligámica o poligínica y con predominio de la familia extensa y la filiación unilineal, operan, al hacerlo, ciertas violencias simbólicas sobre su complejidad, pluralidad y diversidad. En esta labor, el trabajo de campo, la etnografía y, en general, las metodologías cualitativas son herramientas fundamentales, pero si no existe una reflexión previa sobre los conceptos y sus limitaciones - o sobre los sistemas de pensamiento y de aprehensión de la realidad-, no existe una epistemología capaz de abordar un análisis mínimamente serio y profundo del cambio social, cultural, político y económico que está experimentando la sociedad marroquí en la contemporaneidad. Las investigaciones etnográficas, sociológicas y antropológicas que se refieran a Marruecos tienen que contribuir a ofrecer una explicación compleja y bien articulada sobre aspectos múltiples de lo social. En ocasiones, este tipo de investigaciones ejercen un efecto contrario, puesto que ofrecen una fotografía fija de lo que no es sino una cosa en movimiento.

Esto que aquí señalamos a través del ejemplo marroquí también vale para una parte de la bibliografía que se genera en ámbitos académicos de todo el mundo sobre otras sociedades de mayoría musulmana. En muchos trabajos de carácter sociológico y antropológico, no se aplican razonamientos de tipo inductivo (extracción de conclusiones generales a partir de datos particulares), sino básicamente razonamientos de tipo deductivo, con un valor esencialmente demostrativo y reiterativo. La literatura académica que ofrece una visión victimista de las experiencias vitales de las mujeres en las sociedades de mayoría musulmana se reproduce normalmente sobre sí misma, y abusa del monolitismo que parece caracterizar actualmente el uso de ciertos conceptos en ciertos 
discursos sobre lo social. Lamentablemente, cierta bibliografía ha hecho que el concepto de patriarcado se haya convertido en uno de estos conceptos monolíticos, poco sensible para representar las acciones humanas, tanto de hombres como de mujeres. La bibliografía disponible sobre el patriarcado o sobre los sistemas patriarcales es utilizada, en ocasiones, como una fuente de premisas, y ciertos investigadores únicamente se mueven de lo general a lo particular para confirmar la verdad del sistema teórico al que se adscriben de partida. La incidencia de ciertos hechos sociales problemáticos se minimiza, se elimina del discurso o se (re)elabora como «excepción». La relativa ausencia de debate explícito sobre los conceptos se une a la escasez de científicos sociales «en el campo» y da lugar a una bibliografía sociológica y antropológica «de sillón» que dificulta la evaluación de los procesos de transformación social y cultural, el análisis de las relaciones de género y de los roles de las mujeres y los hombres en sociedad, así como el estudio del establecimiento y la perpetuación de las desigualdades sociales y de género, además de las relaciones de dominación y subordinación interpersonales e intercolectivas.

\section{La extensión del concepto de patriarcado y la noción de kiriarquía}

La etimología del término patriarca nos informa sobre el significado del concepto de patriarcado. Este término viene del latín patriarcha y del griego $\pi \alpha \tau \rho$ ió $\rho \chi \eta \varsigma$, un compuesto formado por dos palabras: pater o 'padre' ( $\pi \alpha \tau \eta$ $\rho)$ y arché o 'gobierno' y 'dominio' ( $\alpha \rho \chi \eta ்)$. La palabra patriarca se refiere a la máxima autoridad familiar y a la máxima autoridad política, a un varón que, por su condición de padre (y por su edad), ejerce autoridad en el seno de la familia y sobre otros colectivos. De este modo, la palabra patriarcado (en inglés, patriarchy; en francés, patriarcat) se refiere a un sistema social o de gobierno basado en la autoridad de los hombres de mayor edad o de los hombres que han sido padres —o, más bien, de los varones que tienen hijos, preferentemente varones, que forman una parte importante de su capital simbólico.

La manera en que se generan y se usan una serie de conceptos teóricos en algunos textos de gran difusión que aparecen en la bibliografía sociológica y antropológica contemporánea sobre las sociedades de mayoría musulmana merece un análisis detallado. Por dicha razón, en este artículo, nos proponemos realizar un recorrido por las propuestas teóricas y las conceptualizaciones del patriarcado que mayor influencia ejercen actualmente sobre la forma en que los sociólogos y los antropólogos abordan el estudio de las desigualdades sociales y de género en diferentes sociedades de mayoría musulmana. En el presente artículo, no pretendemos dar cuenta del modo en que se usan los conceptos de patriarcado y androcentrismo en el estudio de las sociedades de mayoría musulmana. Este tipo de investigación excedería la extensión de un artículo, y quizá también la de una o dos monografías. Además, las realidades de las sociedades de mayoría musulmana actuales son muy heterogéneas, tanto si se pretende considerar lo que acontece en distintos colectivos, como lo que acontece en el interior de una de esas sociedades. En realidad, el propósito de 
este texto es el de evaluar algunas de las vías a través de las cuales el concepto de androcentrismo constituye una alternativa al concepto de patriarcado. Por ello, en uno de sus epígrafes, se examinará la génesis y el desarrollo de algunas de las propuestas teóricas más influyentes en la actualidad.

En primer lugar, es necesario recordar que la paternidad, la patrilinealidad, la patrilocalidad, la monogamia y la endogamia son entendidas generalmente como estrategias del patriarcado. Los varones dominan sobre las mujeres y sobre otros varones que no son "patriarcas», sino que están sometidos al "patriarca». Los varones se apropian de la fuerza de trabajo de las mujeres y de estos varones. Las mujeres producen y reproducen para el patriarca y para el patrilinaje, es decir, aportan su fuerza de trabajo y realizan las labores reproductivas, entre otras: la gestación y el parto de los hijos del patriarca, así como también la gestación y el parto de los hijos de otros miembros varones del patrilinaje. La paternidad permite al varón ejercer un control sobre sus hijos legitimado socialmente y a través del sistema de parentesco. La monogamia también refuerza el ejercicio de este control, tanto sobre los hijos como sobre la esposa. La endogamia facilita el control de los patrilinajes sobre sus miembros de sexo femenino. Los patriarcas y los patrilinajes también ejercen derechos de propiedad casi exclusivos sobre la tierra y otros medios de producción. La división sexual del trabajo y los patrones de residencia postnupcial (patrilocalidad) colaboran también en el sometimiento de las mujeres.

La teóloga feminista Elisabeth Schüssler Fiorenza (1992) ha creado un neologismo que se refiere a la cuestión fundamental del «dominio» y que pretende dar cuenta de la forma en que el «dominio» genera desigualdad y subordinación. Es la palabra kyriarchy (kiriarquia). Su etimología remite a los términos griegos kyrios ('dueño') y archein ('gobernar' o 'dominar'). Este concepto se refiere, básicamente, a una relación de dominación interpersonal, de modo que, en el neologismo de Schüssler, no hay ninguna marca de género explícita, en parte quizá porque no se creó para explicar la dominación de los hombres sobre las mujeres o de los patriarcas sobre las mujeres, sobre los hijos y sobre otros varones, sino la dominación que ejerce la Iglesia católica sobre sus fieles. La neutralidad del término es tan solo aparente, pero tal neutralidad intenta subrayar que las distintas formas de dominación son básicamente interseccionales e interpersonales.

La propuesta de Schüssler no vacía de contenido el concepto de patriarcado, pero sí lo convierte en un término que alude sólo a uno de los posibles sistemas de dominación interpersonal, un sistema que combina, de forma específica y básica, las variables género y edad. Así las cosas, cabe preguntarse, inspirados por Schüssler: «Es el patriarcado la principal herramienta de control sobre las mujeres?». Schüssler señala que cualquier persona —independientemente del género o no género al que se adscriba o la adscriban— se sitúa en una posición de dominio con respecto a otras personas. Schüssler emplea de forma deliberada la noción de persona y, por tanto, realiza una conceptualización asexuada y agenerizada del ser, del sujeto o del individuo ¿Cuál es el lugar del «género» en esta propuesta teórica? Schüssler estima que la opresión de género debe de ser 
localizada en una suerte de matriz de formas de opresión. Es decir, no niega que existan formas de opresión ligadas al sistema de sexo o género, pero las integra en un sistema global de opresión de unos seres sobre otros. En cualquier caso, es interesante subrayar que el término kiriarquía no define seres sin poder o seres enteramente sometidos a otros (podríamos recordar aquí las muchas investigaciones sobre las «sociedades patriarcales» que han ofrecido una imagen pasiva de las mujeres o que han invisibilizado los aportes femeninos, que han retratado a las mujeres como receptoras pasivas de la opresión). Este término tampoco invisibiliza las agencias de los colectivos y los individuos más vulnerables. La propia Schüssler se ha encargado de ilustrar el modo en que todas las personas participan activamente en las relaciones de dominación sobre otras personas, el modo en que todo el mundo es dominador y dominado u opresor y oprimido.

\section{La conceptualización del androcentrismo}

La introducción del concepto de androcentrismo (en inglés, androcentrism; en francés, androcentrisme) nos remite a una tradición filosófica que presenta las características siguientes:

1) Interesada en la persona.

2) Preocupada por el análisis del poder, el control y la dominación.

3) Empeñada en desenmascarar - en cierto modo, en el sentido en que plantea Taussig su unmasking unmasking - la subordinación y la subalternidad.

4) Comprometida con la identificación de la desigualdad de género y de los procesos de homogeneización, esencialización, diferenciación y jerarquización.

Los discursos y las prácticas androcéntricos no son siempre fáciles de identificar, ya que se mantienen a base de comisiones y omisiones. No basta con decir que el androcentrismo existe cuando el hombre, lo masculino o la masculinidad son considerados la medida de todas las cosas; cuando las acciones individuales reflejan perspectivas, intereses o valores masculinos; cuando el hombre, lo masculino y la masculinidad son considerados fuente única o primordial de sabiduría y autoridad, o cuando las experiencias masculinas son las preeminentes, las normativas, las imitables, las deseables, etc. Es necesario, también, subrayar el refuerzo que otros factores ofrecen al androcentrismo: representaciones y estereotipos, misoginia, sexismo, machismo, marginación, represión, violencia física y simbólica, etc. La deconstrucción del androcentrismo no es una labor sencilla, pero ello no obsta para que el androcentrismo pueda ser considerado una táctica o una estrategia social de una gran fuerza y permeabilidad intersubjetiva. Por alguna razón, o por algunas razones que no se nos escapan enteramente, pero que tampoco llegaremos a determinar con exactitud - siempre podemos aspirar a nuestras «verdades parciales» (partial truths), como decía Clifford-, el androcentrismo surge históricamente en la especie humana como táctica y como estrategia, como ideología y como recurso ideológico, y ha demostrado una enorme perdurabilidad temporal y una amplia 
extensión geográfica. Es una manera de entender a las personas que permea las cosmologías, las ontologías y las epistemologías.

En las ciencias sociales, el concepto de androcentrismo se utiliza preferentemente para subrayar una serie de problemas epistemológicos, y su generalización en la bibliografía tiene lugar en la década de 1970:

1) La invisibilidad de las mujeres y de las actividades femeninas, así como el escaso interés científico en la visibilización de la participación de las mujeres en todos los órdenes de la existencia humana.

2) La identificación entre los hechos de los varones y los hechos de la humanidad, de una sociedad y de una cultura. En algunos estudios antropológicos, el androcentrismo aparece como una característica del sistema patriarcal, cuando en realidad ocurre más bien lo contrario: el patriarcado - definido como la autoridad de los hombres sobre las mujeres - sólo es posible dentro de un sistema ideológico androcéntrico, en el que dicha autoridad se funda en el principio de la supremacía de lo masculino sobre lo femenino. Es lógico pensar que, desde un punto de vista filosófico y teniendo en cuenta que femenino y masculino son categorías englobantes y quizá un tanto esencialistas y universalistas a la vez, es difícil, si no imposible, distinguir o separar de forma neta qué es lo masculino y qué es lo femenino en una subjetividad o persona dada, o en una sociedad o cultura determinada. La antropología en la que estoy pensando en este artículo no está centrada en la definición de las esencias, sino en los procesos, en concreto, en los de construcción de identidades generizadas $y$, por tanto, las categorías masculino y femenino en las que pienso son altamente contextuales. En mi opinión, la pregunta por el control de unos seres humanos sobre otros y la pregunta por el lugar que unos seres humanos ocupan en relación con otros — que es la pregunta por el poder, pero también la pregunta por la construcción de la persona - nos debería llevar a trabajar en una filosofía del concepto de recurso: recurso ideológico y recurso material. En este sentido, el androcentrismo constituye la movilización y la manipulación de un recurso ideológico, tanto por parte de los hombres como de las mujeres. Es obvio que el androcentrismo no es la causa única de los órdenes de poder existentes, pero tanto las ciencias sociales como las ciencias humanas han ayudado a demostrar que constituye un recurso estructural de primera magnitud. Lyotard define la posmodernidad como la incredulidad frente a las metanarrativas, explicaciones de la cultura humana centradas en un factor predominante, de ahí que, a la vez que postulamos que la deconstrucción del androcentrismo es una labor de crítica discursiva esencial, podemos señalar que una de las debilidades de este discurso sobre el androcentrismo es su afán totalizador. La crítica del androcentrismo como ideología dominante no puede ni debe desligarse de la crítica de otros discursos también totalizadores, como la heteronormatividad o el heterocentrismo y el etnocentrismo.

En la investigación sociológica y antropológica sobre las sociedades de mayoría árabe y musulmana, el androcentrismo se analiza en múltiples áreas. 
Así, en el estudio de la religión, algunas investigadoras se han esforzado por demostrar que estamos frente a un androcentrismo histórico y contextual. La labor de estas investigadoras intenta reconocer, analizar y deslegitimar el androcentrismo. En esa labor de deslegitimación, el androcentrismo debería perder su valor instrumental como recurso, pero esta labor necesita la acción de muchos más agentes que los científicos sociales. En cualquier caso, tanto el concepto de androcentrismo, en relación con lo ideológico, como el concepto de agnatismo, en relación con el parentesco, sirven para articular explicaciones complejas de una forma más amplia y también libre de los reduccionismos que opera el concepto de patriarcado. Es muy importante tener en cuenta que el androcentrismo funciona con frecuencia como sistema predominante de legitimación de la autoridad del "patriarca», y es en este sentido en el que insistiremos, en el último epígrafe de este artículo, en su potencial analítico frente al concepto de patriarcado.

\section{La influencia del concepto de patriarcado en el estudio de las sociedades de mayoría árabe y musulmana}

Las sociedades de mayoría musulmana suelen ser caracterizadas, casi de modo general y absoluto por los especialistas, sociólogos y antropólogos, como sociedades patriarcales. Los estudios feministas y de género utilizan con frecuencia el concepto de patriarcado. Este es un término profundamente anclado en los discursos feministas; por un lado, porque, en buena medida, el feminismo se desarrolla para desafiar al androcentrismo y al sexismo y, por otro, porque lo ha hecho a través de este instrumento teórico de gran eficacia simbólica. Tanto en la bibliografía sociológica y antropológica contemporánea en lengua inglesa, como en la bibliografía en lengua francesa, encontramos múltiples formas de utilización del concepto de patriarcado en relación con las sociedades de mayoría musulmana. Así, en la bibliografía en lengua francesa, algunos conceptos que se repiten con insistencia son los de «sistema patriarcal» (système patriarcal), «estado patriarcal» (état patriarcal), «orden patriarcal» (ordre patriarcal), «valores patriarcales», «ideas patriarcales» $\mathrm{y}$ «discursos patriarcales» (valeurs patriarcales, idées patriarcales y discourses patriarcales) o, incluso, «islam patriarcal».

En este epígrafe, exploraremos algunos ejemplos de la proliferación conceptual reciente en torno a los términos patriarcado y patriarcal, y lo haremos a través de obras que han ejercido una influencia que va más allá del ámbito lingüístico y geográfico en el que se han generado.

En sendos trabajos de 1982 y 1988, John C. Caldwell (1982) y Deniz Kandiyoti (1988) delimitan la extensión geográfica del denominado «cinturón del patriarcado clásico» (belt of classic patriarchy), que incluiría el norte de África (con Marruecos), el Oriente Medio musulmán (con Turquía e Irán) y el sur y el este de Asia (con Pakistán, Afganistán, el norte de la India y la China rural) (Moghadam, 2004: 143). John C. Caldwell, demógrafo, había propuesto el término "cinturón patriarcal» (patriarchal belt) en 1982. Caldwell entendía que se trataba de una región geográfica que comprendía el norte de África y el 
Oriente Medio musulmán (incluía Turquía, Pakistán e Irán), así como el sur y el este de Asia (incluía India y China). En 1985, Deniz Kandiyoti define, a partir del área geográfica mencionada, la existencia de un "patriarcado clásico» (classic patriarchy). En este sistema, que Kandiyoti define a partir de su estudio de la sociedad rural turca, el poder de los hombres viene de la propiedad de la tierra y del control del ámbito doméstico. Los hombres de mayor edad dominan a los hombres jóvenes y a todas las mujeres. Los padres rigen sobre los hijos, y las jerarquías de poder están basadas en la edad, en la generación y en el género. Kandiyoti señala que, en la reproducción de este sistema, tanto la naturaleza cíclica de la subordinación femenina como la relativa autonomía en las vidas de las mujeres son altamente instrumentales (Kandiyoti, 1985: 58). Los autores que han contribuido con mayor fuerza a construir el concepto de patriarcado en este ámbito geográfico tan vasto son Deniz Kandiyoti, Mervat Hatem y Suad Joseph. Deniz Kandiyoti (1988), frente a lo que denomina "patriarcado clásico", acuña el concepto patriarchal bargain (en nuestra traducción, 'negociación o pacto patriarcal'), que indica la existencia de reglas establecidas que regulan las relaciones de género, a las que ambos, mujeres y hombres, se adaptan, aunque puedan ser impugnadas, redefinidas y renegociadas (Kandiyoti, 1988: 286). Este es un concepto, traducido en la idea de que es posible una "negociación con el patriarcado» (bargaining with patriarchy), que ha sido ampliamente utilizado para explicar formas de dominación masculina en sociedades de mayoría musulmana muy distintas. A través de este concepto, Kandiyoti y otros investigadores ponen de relieve que existen formas de respuesta femenina frente al patriarcado, formas que resultan de la subversión o de la adaptación. La perspectiva de Kandiyoti sobre las acciones femeninas en torno al patriarcado renovará el interés por los espacios de contestación en los que actúan las mujeres. Mervat Hatem ha señalado que "clase» y "patriarcado» son dos paradigmas en competencia en el estudio de las mujeres de Oriente Medio (Hatem, 1987) y ha analizado la alianza duradera entre el nacionalismo y el patriarcado en las leyes de estatuto personal en los países musulmanes (Hatem, 1986a), ocupándose específicamente del caso egipcio. En lo que ella denomina «el sistema patriarcal segregado egipcio», también ha trabajado sobre las políticas relativas a la sexualidad y el género en los siglos XVIII y XIX (Hatem, 1986b). En los estudios de Hatem, el patriarcado es un sistema de relaciones estructurales que puede ser revertido a través de la acción individual y también de la acción del estado, pero la socialización de los individuos en este sistema facilita su perpetuación. Mervat Hatem subraya que las características del patriarcado deben ser discutidas en su contexto histórico específico, y que es necesario explorar en qué medida difieren unos y otros sistemas patriarcales, tanto desde el punto de vista diacrónico como sincrónico. Es interesante notar que los estudios de Mervat Hatem, centrados en el caso egipcio, han tenido un notable impacto en el análisis de muchas sociedades de mayoría árabe y musulmana. De hecho, en uno de sus textos más citados, ella misma considera que "clase» $\mathrm{y}$ "patriarcado» son dos paradigmas en el estudio de las mujeres de Oriente Medio (Hatem, 1987). 
Suad Joseph $(1993,1994,1996)$ define el patriarcado en el contexto árabe como la priorización de los derechos de los hombres y de los mayores (incluyendo a las mujeres mayores) y la justificación de esos derechos dentro de los valores del parentesco normalmente sostenidos por la religión. Joseph multiplica los conceptos y habla de un "patriarcado político», un "patriarcado económico» y un "patriarcado religioso», a la vez que analiza la internalización del patriarcado por el yo (Joseph, 1996). En su estudio sobre la sociedad urbana libanesa, Joseph también ha propuesto el concepto de «conectividad patriarcal» (patriarchal connectivity) (Joseph, 1993). Joseph indica que este concepto se refiere a la producción de subjetividades con fronteras fluidas organizadas para la dominación de género y de edad en una cultura que valora las estructuras de parentesco y la moralidad (Joseph, 2001: 24).

La definición de Knauss (1987: XII) es la de un precursor, ya que entiende el patriarcado como «una jerarquía de autoridad que está controlada y dominada por los hombres» y que tiene su origen en la familia. Los trabajos de Knauss se refieren a la sociedad argelina del siglo XX y preludian los análisis llevados a cabo en Oriente Medio por Suad Joseph.

Las críticas realizadas al concepto de patriarcado tal y como lo formulan estos cuatro autores mencionados son múltiples y, en algunos casos, son anteriores a la exitosa exportación de este concepto desde el feminismo de Occidente al de Oriente (aquí simplifico en los términos geopolíticos, pero no en la dirección del movimiento). Sylvia Walby, en 1989, propone una nueva forma de teorizar el patriarcado, al que considera una herramienta esencial en el análisis de las relaciones de género. La mayor parte de los autores incómodos con el concepto de patriarcado subrayan su ahistoricismo y su falsa universalidad, pero Walby sugiere que, no obstante estas puntualizaciones, pueden identificarse ciertas formas de patriarcado históricas y específicas, y se pueden analizar proponiendo una idea del patriarcado más flexible que sirva para estudiar las continuidades y las formas de desigualdad de género variables históricamente y desde un punto de vista cross-cultural (Walby, 1989: 229-230). La crítica de Shibles (1991) es más incisiva, en tanto que trata de demostrar que el patriarcado es un "mito» y cuestiona la utilización del patriarcado como causa única de fenómenos multicausales.

La bibliografía feminista y buena parte de los estudios de/sobre el género, en particular los centrados en las relaciones de género, utilizan el concepto de patriarcado con profusión y con resultado desigual. La utilización acrítica del concepto ha generado muy poca reflexión hasta la fecha, pero, junto con las mencionadas críticas de Walby (1989) y Shibles (1991), en este artículo, me gustaría recoger algunas otras aportaciones que cuestionan el concepto y que considero especialmente interesantes. Las reflexiones proceden del llamado "feminismo de segunda ola» o "segunda ola del feminismo" (second wave feminism) y subrayan que la utilización acrítica del concepto de patriarcado genera perspectivas simplistas y distorsionadas sobre realidades complejas, y oculta acciones femeninas que necesitan mayor visibilidad práctica: agencia y autonomía, pero también estrategia y táctica. Es necesario, de acuerdo con 
estas investigadoras, revelar la ambivalencia del concepto (Beechey, 1979) y repensar su pertinencia como categoría o sistema de análisis (Acker, 1989; Pollert, 1996).

En 1996, Anna Pollert publica un artículo en el que formula una crítica bastante consistente del uso actual del concepto de patriarcado. En las últimas décadas, este concepto ha sido empleado preferentemente como herramienta de análisis de las desigualdades de género, pero, de acuerdo con Pollert, el uso simultáneo del concepto para ofrecer una descripción y una explicación resulta un tanto problemático. La teoría del patriarcado acusa una notable circularidad y ciertos reduccionismos, y su aplicación suele ser mecánica. La propuesta de Pollert se encuadra dentro del materialismo histórico feminista y sigue la estela de varias investigadoras preocupadas por la intersección del género, la clase y el trabajo. Lo interesante del artículo de Pollert es que intenta explicar la fuerza que tiene el patriarcado como metanarrativa, o como grand narrative en la actualidad, una cuestión que, indudablemente, le concede un gran valor discursivo, pero le resta valor analítico. Heidi Gottfried (1998) examina la crítica de Pollert y propone saludar cualquier intento de desarrollar la teoría más allá del concepto de patriarcado, centrando la investigación en los cuerpos, en los espacios y en las experiencias generizados (gendered):

An excavation of lived practices can make visible the gendering process and ground analysis of specific forms of male power in relationship to class and other hierarchies. Thus, excavation makes visible these taken-for-granted assumptions orienting practical activity and thus can make them available for feminist criticism and action. (Gottfried, 1998: 465)

Esta mirada procesual en torno al patriarcado ha sido desarrollada en muchos trabajos sociológicos y antropológicos que analizan la forma en que se transmite la ideología de la dominación de los hombres sobre las mujeres, tanto intergeneracionalmente como a través de la comunicación social. En este sentido, el estudio de los procesos de socialización y enculturación deviene básico.

En la bibliografía sobre las sociedades de mayoría musulmana, otras propuestas teóricas amplían el concepto de patriarcado para dar cuenta de su dinamismo. Esto ocurre fundamentalmente en la década de $1990 \mathrm{y}$ en la primera del 2000. Hisham Sharabi (1988) habla de "neopatriarcado»; Riffat Hassan (1991), de "postpatriarcado", y Valentine Moghadam (2004), de "patriarcado en transición». La expresión neopatriarcado cuenta con cierto predicamento en la investigación sobre la política en los países árabes, singularmente en los trabajos de E. Haghighat sobre la fuerza de trabajo femenina en países musulmanes. Sharabi se refiere, con tal concepto, a los survivals de un patriarcado tradicional y precapitalista en las sociedades árabes que han experimentado procesos históricos de modernización. Sharabi habla de un «estado neopatriarcal» que, en cierto sentido, «no sería más que una versión modernizada del tradicional sultanato patriarcal» (Sharabi, 1988: 46). La idea general que sostiene Sharabi es que, en el último siglo, las estructuras patriarcales de las sociedades árabes no se han desplazado o se han modernizado verdaderamente, sino que 
se han fortalecido y han permanecido en formas más o menos deformadas y «modernizadas» (Sharabi, 1988: 4). Sharabi se refiere a las sociedades de mayoría árabe y, por tanto, sus análisis incluyen reflexiones sobre lo que acontece en veintiún estados soberanos, desde el Atlántico hasta el Golfo Pérsico, y sobre las prácticas de doscientos millones de personas. Sharabi establece una verdadera teoría social cuando expresa que los cambios económicos, sociales, políticos y culturales que han experimentado las sociedades árabes en el último siglo no han conducido a una modernidad, sino a un neopatriarcado. En este sentido, Sharabi considera que el fundamentalismo religioso es una ideología propia de este neopatriarcado.

La expresión "postpatriarcado» que utiliza Riffat Hassan es una utopía de referencia para cierta investigación feminista (Lentin, 1995, por ejemplo, en su citado lema "I'll be a post-feminist in post-patriarchy», secundado por Schwenken y Ullrich (2003), con su «We'll be post-feminists in post-patriarchy»), pero en el sentido en que la usa esta investigadora se refiere fundamentalmente a un islam postpatriarcal, un islam que se construye en la contemporaneidad a partir de una fuerte crítica (y rechazo) de los postulados del patriarcado. En concreto, Hassan, teóloga, feminista y experta en el islam, habla de un islam liberado de las interpretaciones teológicas en clave patriarcal.

Moghadam utiliza el término patriarcado en sus estudios sobre Iraq, Irán y Afganistán. La idea de un "patriarcado en transición» la expone, en 2004, en un artículo sobre las mujeres del área MENA (Middle East and North Africa; Oriente Medio y el norte de África) y sobre los cambios que se han producido en las últimas décadas en la familia musulmana. Moghadam asume las definiciones de Kandiyoti y Sharabi («patriarcado clásico» y «neopatriarcado»), pero acentúa la idea de transición, que, en este contexto, constituye su aportación más novedosa.

En párrafos previos, hemos mencionado a Riffat Hassan y la hemos definido como experta en feminismo e islam. De hecho, Riffat Hassan es una de las primeras investigadoras que asume escribir desde el «feminismo islámico» (Hassan, 2001). En este punto del artículo, conviene que exploremos algunos usos del concepto de patriarcado por parte de autoras que se adscriben a la corriente intelectual y sociopolítica denominada "feminismo islámico». A pesar de las profundas controversias que genera la definición de dicha denominación, existe cierto consenso en torno a las propuestas que se realizan en su nombre, propuestas encaminadas a la consecución de una justicia de género en un contexto islámico. En el seno de este movimiento, como han explicado repetidamente Margot Badran o Valentine Moghadam (Badran, 2006; Badran, 2010, y Moghadam, 2002), investigadoras que mantienen líneas de investigación diversas, como Asma Barlas, Amina Wadud, Ziba Mir-Hosseini, Aziza al-Hibri o Leila Ahmed, han utilizado generosamente el concepto de patriarcado, tanto para pensar un orden social más justo, más allá de las formas de dominación que instituye este sistema (precisamente, uno de los artículos más sobresalientes de Wadud se titula «Beyond patriarchy»), como para estudiar la forma en que este patriarcado ha calado en los discursos y en las prácticas 
religiosas musulmanas. En muchas de las contribuciones que se encuentran en la órbita del llamado "feminismo islámico», las investigadoras se han preguntado qué hay "después del patriarcado» (after patriarchy), considerando el patriarcado como un lapso temporal, un tiempo histórico, y «más allá del patriarcado" (beyond patriarchy), considerando el patriarcado como un orden social y político determinado.

Lógicamente, la cuestión de fondo a la que remiten los conceptos hasta ahora mencionados es la existencia de jerarquías y de desigualdades entre personas de sexo o género diferente. De forma básica, todos los trabajos citados hasta el momento intentan ofrecer una explicación a la existencia de jerarquías, así como a la existencia de desigualdades sociales, de desigualdades políticas y de desigualdades fundamentadas en el sistema de sexo y género.

En las formulaciones sucesivas del concepto de patriarcado y en las críticas sucesivas, varios investigadores han señalado que la historiografía permanece articulada en torno a demasiados opuestos: empancipación/no liberación (Kandiyoti, 1988); controladas/autónomas (Shaheed, 1994); de ahí que se haya intentado superar o transgredir esta normatividad dicotómica a través de conceptos auxiliares como el de negociación (negotiation, bargain). Así, la propia Kandiyoti (1988) habla de una «negociación con el patriarcado" (bargaining with patriarchy, patriarchal bargaining); Safi (2000), de una «negociación con la baraka»; Rosen (1984), "con la realidad" (bargaining for reality), y Alon (1997), de una «negociación con Dios» (bargaining with God). La propuesta teórica de Kandiyoti, a la que algunos califican de «modelo», ha tenido un eco relativo en los estudios sobre el parentesco: Lorber y Bandlamundi (1993) hablan de "negociación patriarcal» (patriarchal bargaining) y especifican, dentro de su análisis de la infertilidad masculina, acciones que constituirían una «negociación marital» (marital bargaining); Hutson, en 2001, habla de una «negociación patriarcal» o «del patriarcado» (patriarchal bargaining) para referirse a las relaciones entre hombres y mujeres dentro de la orden sufí Tijaniyya en Nigeria, y Yount (2005), para analizar ciertos aspectos de la corresidencia o de lo doméstico en Egipto (la violencia doméstica y el poder doméstico, singularmente). Igualmente, se habla de «negociación de la identidad» (Bartkowski y Read, 2000), de "negociación de la identidad femenina musulmana» (Khan, 2002), de «negociación de la fe» (Al-Yousuf, 2006) y de «negociación de las diferencias» (Dwyer, 1999), fundamentalmente en estudios sobre la llamada «interculturalidad» o «multiculturalidad». La distinción entre lo público y lo privado tiene también una gran significación en los estudios sobre las mujeres, sobre el parentesco y sobre el islam. En este momento la mencionamos porque constituye un buen ejemplo de la manera en que la sociología y la antropología trabajan con simplificaciones dicotómicas. Joseph (1997) habla de la distinción entre lo público y lo privado como una "frontera imaginada» (imagined boundary); Hefner (2001) habla de un «islam público», al igual que Salvatore y Eickelman (2004). También hay estudios que postulan que es posible «reordenar» lo público y lo privado (Graham y Khosravi, 2002), y, aceptando esta dicotomía, Eickelman y Anderson (2003) hablan de la emergencia de la 
«esfera pública»; Anderson (2003), de la «reconfiguración de la esfera pública», y Esposito y Burgat (2003), de la presencia de la religión en la esfera pública.

\section{Androcentrismo, ¿̨alternativa conceptual?, ¿̨herramienta de visibilización femenina?}

En las primeras páginas de este artículo, remitíamos a la pregunta planteada por Yolanda Aixelà (2000) ("ipatriarcalidad o androcentrismo?»), en un trabajo que habla por extenso sobre la existencia de desigualdades sociales en una sociedad de mayoría musulmana: Marruecos. A partir de ahí, evaluábamos algunas de las principales propuestas teóricas formuladas en torno al concepto de patriarcado en estudios sociológicos y antropológicos de sociedades de mayoría musulmana publicados en las tres últimas décadas. Estas propuestas han sido generadas a través del análisis de sociedades heterogéneas, pero han sido aplicadas por muchos investigadores sociales en numerosos contextos de lo que se ha dado en llamar «mundo musulmán», contextos distintos de aquellos que pretendían explicar en origen. En el presente epígrafe, intentaremos explorar qué aporta, de forma básica, el término androcentrismo frente al término patriarcado, tanto si se lo considera una herramienta heurística que permite profundizar en el conocimiento de la dominación interpersonal e intercolectiva, como si se tiene en cuenta su versatilidad.

La primera vez que se utiliza el término androcentrismo es en la obra de Charlotte Perkins Gilman (1911) titulada The Man-Made World or our androcentric culture. En ella, la autora describe una cultura centrada en el varón o un sistema de pensamiento en el cual lo relativo al varón es identificado o valorado como lo normativo. Es evidente que, desde esa fecha, el concepto se ha aplicado a fenómenos sociales muy diversos. En los estudios sociológicos y antropológicos sobre las sociedades de mayoría musulmana que hablan de androcentrismo, el término permite explicar fenómenos que no están relacionados únicamente con la cuestión de la «autoridad» o el «poder» que ejercen unas personas sobre otras, hombres sobre mujeres u hombres de edad mayor sobre mujeres y hombres de edad menor. El concepto de androcentrismo es, ya de partida, un concepto que remite a un fenómeno más amplio que el de patriarcado. Un concepto que se refiere a las cosmologías, a las ontologías y a las epistemologías. Numerosos trabajos ponen de relieve la fuerza fundacional del androcentrismo, sobre el que se sustenta con enorme frecuencia la condición preeminente del varón. La existencia de un sistema patriarcal o de un orden patriarcal no puede ser explicada en términos absolutos. Es necesario profundizar en los discursos y en las prácticas que legitiman este hipotético sistema o este hipotético orden, así como también en aquellos discursos y prácticas que revelan las situaciones plurales de las mujeres y de los hombres en el interior de sociedades preeminentemente androcéntricas.

Frente a la relativa proliferación conceptual en relación con el concepto de patriarcado, el concepto de androcentrismo aparece en los estudios sociológicos y antropológicos sobre sociedades de mayoría musulmana publicados 
en las tres últimas décadas como un concepto que aún no se ha explorado en profundidad. Esto se traduce en que existen numerosos estudios sobre el androcentrismo en el lenguaje, en la enculturación, en la educación y en la formación de la persona, así como en la religión (el islam), pero menos estudios que evalúen el androcentrismo de las instituciones y de las prácticas sociales.

La hipotética universalidad del androcentrismo que asumen muchos investigadores sociales haría difícil, si no imposible, dirimir si el androcentrismo de las sociedades de mayoría musulmana posee un origen islámico o anteislámico, una cuestión que se plantea con relativa frecuencia en la bibliografía. De hecho, esta es una de las cuestiones que surgen al evaluar el androcentrismo de las religiones monoteístas, entre ellas el islam, y al examinar en perspectiva histórica las realidades sociales de hombres y mujeres. Una de las líneas de investigación más fértiles sería la que cruza el análisis del androcentrismo con el estudio de otros recursos ideológicos sobre los que descansan las relaciones de dominación interpersonales e intercolectivas, como el sexismo, la misoginia, el racismo, el clasismo, etc. En las investigaciones sobre lo religioso, que tienen una importancia fundamental, el estudio del androcentrismo se suele plantear en conexión con el estudio del teocentrismo y con el examen de la exégesis textual. Esta es, sin duda, una de las líneas de investigación más prometedoras en el interior de la corriente del feminismo islámico.

A modo de conclusión, podemos subrayar que, desde las décadas de 1980 y 1990, sobre todo en los estudios feministas y de género, múltiples críticas al concepto de androcentrismo han señalado que existen notables «verdades parciales» en el conocimiento generado desde estos presupuestos. Por un lado, porque resulta complicado asumir que existan formas de feminidad distintas de las formas de masculinidad y, por otro, porque los estudios sobre las masculinidades en las sociedades de mayoría musulmana se encuentran en un estadio incipiente, y es evidente que no es posible hablar de una noción unitaria de hombre, varón o de lo masculino en un ámbito tan diverso. Es por ello por lo que, retomando los argumentos que expresábamos en el primer epígrafe de este artículo, convendría que los sociólogos y los antropólogos insistiéramos en la necesidad de intensificar los acercamientos etnográficos a las sociedades sobre las que escribimos, así como que subrayáramos la conveniencia de promover la elaboración de conceptos heurísticos y epistemológicos a partir de lo observado en estos contextos locales.

\section{Referencias bibliográficas}

ACKER, J. (1989). «The problem with patriarchy». Sociology, 23, 235-240.

Aixelà CABRÉ, Y. (2000). Mujeres en Marruecos: Un análisis desde el parentesco y el género. Barcelona: Edicions Bellaterra.

AL-Yousuf, H. (2006). «Negotiating faith and identity in Muslim-Christian marriages in Britain». Islam and Christian-Muslim Relations, 17 (3), 317-329.

Alon, I. (1997). «Bargaining with God». Le Muséon, 110 (1-2), 223-247.

ANDERSON, J. W. (2003). «New media, new publics reconfiguring the public sphere of Islam». Social Research, 70 (3), 887-906. 
BADRAN, M. (1985). «Islam, patriarchy, and feminism in the Middle East». Trends in History, 4 (1), 49-71.

- (2006). «Islamic feminism revisited». Al-Akhram Weekly, 781, s. p.

- (2010). «Où en est le feminism islamique?». Critique Internationale, 46, 25-44.

Bartkowsky, J. P. y Read, J. G. (2000). "To veil or not to veil?: A case study of identity negotiation among muslim women in Austin, Texas». Gender and Society, $14(3), 395-417$.

Beechey, V. (1979). «On Patriarchy». Feminist Review, 3, 66-82.

Caldwell, J. (1982). Theory of fertility decline. Londres: Academic Press.

DWYER, C. (1999). "Veiled meanings: Young British muslim women and the negotiation of differences». Gender, Place and Culture, 6 (1), 5-26.

Eickelman, D. F. y Anderson, J. W. (2003). New Media in the Muslim World: The Emerging Public Sphere. Bloomington: Indiana Series in Middle East Studies.

Esposito, J. L. y Burgat, F. (2003). Modernizing Islam: Religion in the public sphere in Europe and the Middle East. Londres: Hurst.

GotTfried, H. (1998). «Beyond Patriarchy: Theorizing Gender and Class». Sociology, $32(3), 451-468$.

GraHAM, M. y KHOSRAVI, S. (2002). «Reordering public and private in Iranian cyberspace: identity, politics and mobilization». Identities: Global Studies in Power and Culture, 9 (2), 219-246.

Haghighat, E. (2005). «A Comparative Analysis of Neopatriarchy and Female Labor Force Participation in Islamic Countries». Electronic Journal of Sociology. <http:// www.sociology.org/content/2005/tier1/_islamLaborforce.pdf>

HASSAN, R. (1991). "Muslim women and post-patriarchal Islam». En: CoOEY, P. M.; Eakin, W. R. y McDaniel, J. B. (eds.). After Patriarchy. Feminist Theory of the World Religions. Maryknoll, Nueva York: Orbis Books.

- (2001). "Challenging the stereotypes of fundamentalism: An Islamic feminist perspective». The Muslim World, 91 (1-2), 55-70.

Hatem, M. (1986a). "The enduring alliance of Nationalism and Patriarchy: The case of Modern Egypt». Feminist Issues, 6 (1), 20-43.

- (1986b). "The politics of sexuality and gender in segregated patriarchal systems: The case of 18th and 19th century Egypt». Feminist Studies, 12, 251-274.

- (1987). "Class and patriarchy as competing paradigms for the study of Middle Eastern women». Comparative Studies on Society and History, 29 (4), 811-818.

HefNer, R. W. (2001). «Public Islam and the problem of democratization». Sociology of Religion, 62 (4), 491-514.

HuTSON, A. S. (2001). "Women, men and patriarchal bargaining in an Islamic sufi order: The Tijaniyya in Kano, Nigeria, 1937 to the present». Gender and Society, 15 (5), 734-753.

JOSEPH, S. (1993). "Connectivity and patriarchy among urban working class families en Lebanon». Ethos, 21 (4).

- (1994). «Brother/sister relationships: Connectivity, love and power in the reproduction of Arab patriarchy». American Ethnologist, 21 (1), 50-73.

- (1996). "Patriarchy and development in the Arab world». Gender and Development, 4 (2), 14-19.

- (1997). «The public/private: The imagined boundary in the imagined nation/state community: The Lebanese case». Feminist Review, 57, 73-92.

- (2001). Gender and Citizenship in the Middle East. Nueva York, Syracuse: University Press. 
KandiYOTI, D. (1985). Women in rural production systems: Products and policies. París: UNESCO.

- (1988). «Bargaining with patriarchy». Gender and Society, 2 (3), 274-290.

KHAN, S. (2002). Aversion and desire: Negotiating muslim female identity in the Diaspora. Toronto: Women's Press.

KNAUSS, P. S. (1987). The persistence of patriarchy: Class, gender and ideology in twentieth century Algeria. New York: Praeger publishers.

LENTIN, R. (1995). I'll be a post-feminist in post-patriarchy: Reflexivity is a feminist issue [en línea]. Dublín: Department of Sociology. Trinity College. <http://ireland.iol. $\mathrm{ie} / \sim$ mazzoldi/toolsforchange/postmet/feminist.html>.

LORBER, J. y BANDLAMUNDI, L. (1993). "The dynamics of marital bargaining in male infertility». Gender and Society, 7 (1), 32-49.

MOGHADAM, V. M. (2004). "Patriarchy in transition: women and the changing family in the Middle East». Journal of Comparative Family Studies, 35 (2), 137-162.

- (2002). «Islamic feminism and its descontents: toward a resolution of the debate». Signs, 27 (4), 1135-1171.

POllert, A. (1996). "Gender and class revisited; or, the poverty of "Patriarchy"». Sociology, 30 (4), 639-659.

Rosen, L. (1984). Bargaining for reality: The construction of social relations in a Muslim community. Chicago: The University of Chicago Press.

SAFI, O. (2000). "Bargaining with baraka: Persian Sufism, "Mysticism”, and Premodern politics». The Muslim World, 90, 259-287.

Salvatore, A. y Eickelman, D. F. (2004). Public Islam and the common good. Leiden: Brill Publishers.

SCHÜSSLER FIORENZA, E. (1992). But She Said: Feminist Practices of Biblical Interpretation. Boston: Beacon.

SCHWENKEN, H. y UllRich, C. (2003). "We'll be post-feminists in post-patriarchy: Doing feminism between theoretical concepts and political strategies». Comunicación presentada en la Workshop Feminist politics in the 21st century. Theoretical concepts, Political strategies. Universidad de Dortmund, 4 de junio.

SHAHEED, F. (1994). "Controlled or autonomous: Identity and the experience of the network, Women living under muslim laws». Signs, 19 (4), 997-1019.

Sharabi, H. (1988). Neopatriarchy: A theory of distorted change in Arab society. Nueva York: Oxford University Press.

SHibles, W. (1991). "The myth of patriarchy». The Journal of Value Inquiry, 25, 305-318.

WALBY, S. (1989). «Theorising patriarchy». Sociology, 23 (2), 213-234.

YounT, K. M. (2005). «Women's family power and gender preference in Minya, Egypt». Journal of Marriage and Family, 67 (2), 410-428. 